\title{
A Rapid Fatigue Analysis Method of Aluminum Honeycomb Structure with Defects Based on Static Test and Numerical Simulation
}

\author{
Zhao Qian', Ge Meng', Zhang Shunjia ${ }^{1}$ \\ ${ }^{1}$ Shanghai Institute of Mechanical and Electrical Engineering, Shanghai, China \\ ${ }^{2}$ Department of Aerospace Engineering, Harbin Institute of Technology, Harbin, China
}

Email address:

a9b8@qq.com (Zhao Qian), gem_hit@foxmail.com (Ge Meng), parloson1988@sina.com (Zhang Shunjia)

To cite this article:

Zhao Qian, Ge Meng, Zhang Shunjia. A Rapid Fatigue Analysis Method of Aluminum Honeycomb Structure with Defects Based on Static Test and Numerical Simulation. Science Discovery. Vol. 7, No. 5, 2019, pp. 365-372 doi: 10.11648/j.sd.20190705.28

Received: November 9, 2019; Accepted: November 25, 2019; Published: December 3, 2019

\begin{abstract}
At present, most of the substrates of the multifunctional components are honeycomb sandwich structures. Therefore, the study on the fatigue characteristics of honeycomb sandwich structures with defects is helpful to accelerate the engineering application process of the multifunctional structures.In order to study the fatigue characteristics of multi-functional structure under three conditions of tension, compression and three-point bending, the author of this paper proposes a rapid fatigue analysis method of based on statics experiment and numerical simulation.Firstly, the static experiments of the structure under three mechanical conditions of tension, compression and three-point bending are carried out, and then the static simulation is carried out with ANSYS software under the same conditions, and the finite element model in ANSYS is modified according to the experimental results. Finally, the fatigue of the aluminum honeycomb sandwich structure with defects under three conditions is calculated quickly by the fatigue analysis tool in ANSYS The analytical expression between the fatigue life and the load level of the structure under different load ratios is obtained by using the least square method.The fatigue analysis method avoids the complicated fatigue experiments, solves the problem of obtaining the fatigue characteristics of the honeycomb sandwich structure with defects at low cost and high speed under different mechanical conditions and greatly improves the analysis efficiency of the fatigue characteristics of the complex structure.
\end{abstract}

Keywords: Aluminum Honeycomb Sandwich Structure, Static Mechanical Test, Fatigue Simulation, Lattice Defect, Fatigue Life Curve

\section{一种基于静力学试验与数值仿真相结合的含缺陷铝蜂窝结构的快 速疲劳分析方法}

\author{
赵钱 ${ }^{1}$, 葛萌 ${ }^{2}$, 张顺家 ${ }^{1}$ \\ ${ }^{1}$ 上海机电工程研究所, 上海, 中国 \\ ${ }^{2}$ 哈尔滨工业大学航天学院航天工程系, 哈尔滨, 中国
}

邮箱

a9b8@qq.com（赵钱）, gem_hit@foxmail.com(葛萌), parloson1988@sina.com（张顺家）

摘要: 目前, 大部分多功能结构件的基板都是蜂窝三明治结构, 因此, 研究含缺陷蜂窝三明治结构的疲劳特性有助于 加快多功能结构的工程应用进程。为了深入研究多功能结构分别在拉伸、压缩、三点弯曲三种工况下的疲劳特性, 本 文针对含有芯格缺陷的蜂窝三明治结构, 提出了一种基于静力学试验与数值仿真相结合的快速疲劳分析方法, 即先对 
结构进行拉伸、压缩和三点弯曲三种力学工况下的静力学实验, 然后在相同条件下利用ANSYS软件对其进行静力学仿 真, 并根据实验结果修正ANSYS中的有限元模型, 最后在此基础上利用ANSYS中的疲劳分析工具快速计算得到含缺 陷铝蜂窝三明治结构在三种工况下的疲劳特性, 并用最小二乘法得到不同载荷比条件下结构的疲劳寿命与其所受载荷 水平之间的解析表达式。本文提出的疲劳分析方法巧妙地避开了繁琐复杂的疲劳实验, 解决了低成本快速获取含缺陷 蜂窝三明治结构在不同力学工况下疲劳特性的问题, 极大地提高了对复杂结构疲劳特性的分析效率。

关键词: 铝蜂窝三明治结构, 静力学试验, 疲劳仿真, 芯格缺陷, 疲劳寿命曲线

\section{1. 引言}

减小航天器的质量、缩小航天器的体积、降低航天器 的成本是现代航天技术的三大重要目标, 具体来说就是把 航天器的质量、体积以及成本在现有基础上降低一个数量 级, 使我国的航天技术取得重大突破 [1]。航天器内部电子 设备的连接器、电缆、壳体等附加质量约占其总质量的 $50 \%[2,3]$ 。为了能大幅减少这些附加质量, 可以采用多功 能结构技术 [4], 即通过在结构件内部添加芯片来拓展结构 件的功能和用途。而含缺陷蜂窝三明治结构作为一种常见 的低成本多功能结构基板, 快速分析其疲劳特性对多功能 结构技术的工程应用至关重要。

蜂窝三明治结构的疲劳分析方法主要有两种: 疲劳 试验法和有限元仿真法。用试验法来分析疲劳特性的优 点是结果准确, 可信度高。但疲劳试验需要历经结构的 整个疲劳破坏过程, 费时费力, 而且还要进行实时监视, 成本很高。用有限元软件来分析疲劳特性的优点是省时 省力, 成本低, 方便快捷。但由于有限元软件在仿真计 算时作了大量理想化的假设, 而实际的结构由于加工等 因素并非软件仿真中的理想情况, 因此与真实结果相比, 仿真结果的误差较大、可信度较低。国外学者对蜂窝三 明治结构的疲劳问题已经作了大量的研究。2007年,

Belingardi等 [5]探究了缺陷对蜂窝三明治结构 (蜂窝芯子 为铝材, 面板为碳纤维材料) 的四点弯曲特性以及相应 的疲劳性能的影响。他们认为原来的模型需要根据不同 种类的缺陷进行相应的改进。2009年, S.Belouettar等 [6] 研究了两种铝蜂窝三明治结构 (芳纶纤维蜂窝芯子和铝 蜂窝芯子）的四点弯曲工况的疲劳特性。同年, Soni SM 等[7]在低温下对复合材料三明治结构进行了四点弯曲试 验, 研究了其低温疲劳失效形式。2010年, 哈尔滨工业 大学的孔祥皓 [8]对含缺陷的金属蜂窝结构的失效评估与 力学性能进行了研究。其中, 他在研究含有面芯脱焊缺 陷的蜂窝结构时发现在侧拉载荷下的破坏模式与芯子脱 焊结构的结果相近, 而在平拉时, 蜂窝芯子的承载面积 减少, 并会出现应力集中的现象。2013年, Costantino Menna等[9]对蜂窝三明治板进行了结构设计、等效理论 计算、有限元数值仿真、力学试验, 并研究了其破坏变 形形式、减振特性等。同年, Figueroa E等[10]在引入海 水和空气的条件下, 对复合材料泡沫三明治板进行了蠕 变实验和循环加载实验。哈尔滨工程大学的赵鹏成 [11] 提出了用含缺陷蜂窝三明治结构的静强度修正系数 $\mathrm{\epsilon}$ 来 修正完整蜂窝三明治结构的疲劳寿命方程的方法, 巧妙 地避开了繁琐的疲劳实验, 取得了良好的效果。但他所
做的假设过于理想化, 难以用于工程实际。2016年, 哈 尔滨工业大学的柴子龙 [12]在利用有限元法来对蜂窝夹 芯板的疲劳寿命进行预测时, 通过在静态试验中测量的 挠度与有限元软件的计算结果进行比较来验证有限元模 型的正确性, 并在此基础上计算了疲劳寿命, 所得结果 与疲劳试验基本一致。2017年, 王琦等[13]对预埋有面芯 脱焊缺陷的蜂窝板, 在不同的缺陷形状、大小、位置及 蜂窝芯取向的条件下, 均进行了三点弯曲试验。他们发 现缺陷形状对三点弯曲的破坏模式影响很小, 且在相同 的缺陷面积条件下, 纵向弯曲比横向弯曲具有更高的极 限载荷。2019年, 马铭泽等[14]从疲劳试验、失效模式及 损伤发展历程、疲劳寿命预测方法、疲劳曲线规律几个 方面全面介绍了蜂窝夹芯板疲劳研究的最新情况, 同时 也指出了下一步研究的三个方向: 蜂窝纸与蜂窝夹芯板 疲劳特性之间的关系、蜂窝芯内部的损伤发展历程、各 级载荷谱及加载顺序对疲劳寿命累积损伤的影响。

本文针对内部含有芯格缺陷（在蜂窝上面板打孔，去 掉内部的部分蜂窝芯而形成的缺陷）的蜂窝三明治结构, 提出了一种基于静力学试验与数值仿真相结合的快速疲 劳特性分析方法, 即先进行静力学实验, 再利用ANSYS 软件进行相同条件下的静力学仿真, 并根据实验结果修正 有限元模型, 最后在此基础上利用ANSYS软件中的疲劳 工具快速分析结构的疲劳特性。

\section{2. 含缺陷试件在三种典型工况下的静力学实验}

\section{1. 实验目的}

静力学实验是为了获取含缺陷试件在拉伸、压缩、三 点弯曲三种典型工况下线性阶段的位移载荷数据, 为含缺 陷试件有限元模型的搭建提供输入条件, 为后续的疲劳仿 真分析奠定基础。

\section{2. 实验过程及结果}

\subsection{1. 面内拉伸}

用夹具夹紧试件两端的铝块, 然后打开材料试验机开 关, 对试件两端施加拉伸载荷, 同时, 用计算机采集位移 载荷数据, 直到试件被拉断为止。所得到的四条位移-载 荷曲线如下图1所示。试件发生失效时的试验结果照片如 图2所示。 


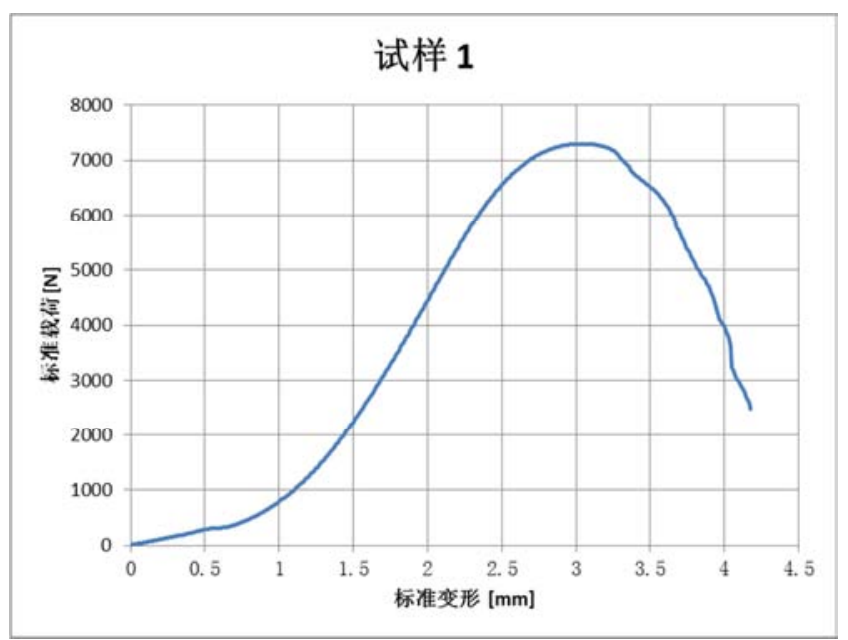

(a)试件1

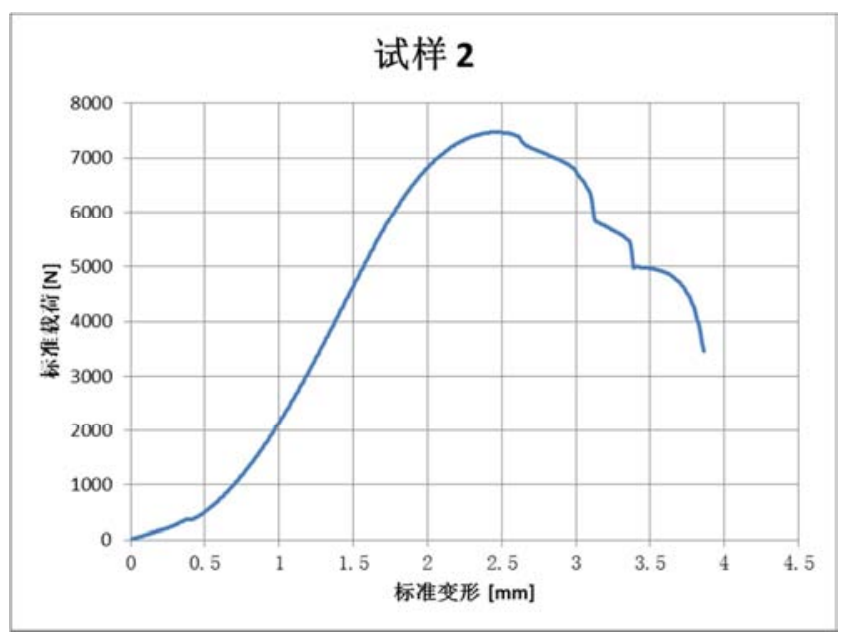

(b)试件2

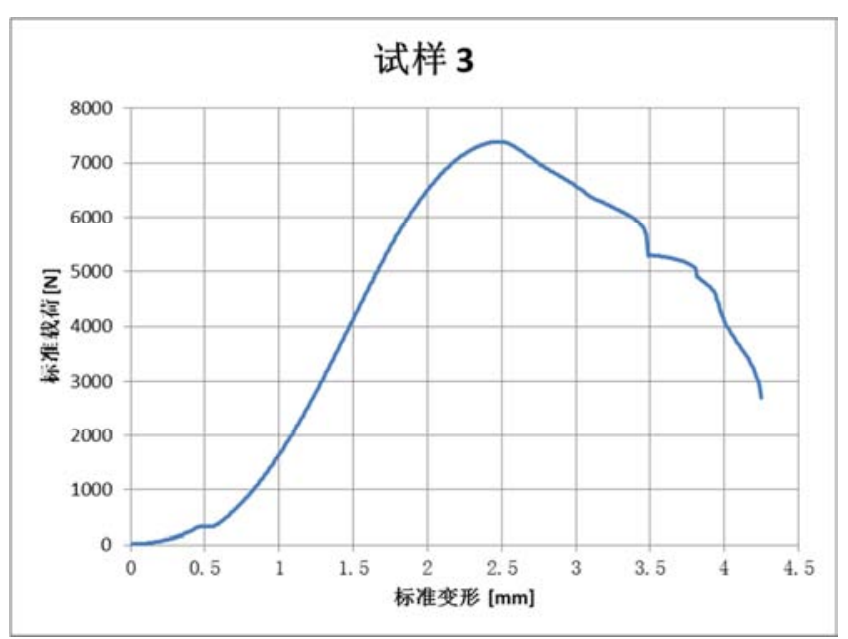

(c)试件3

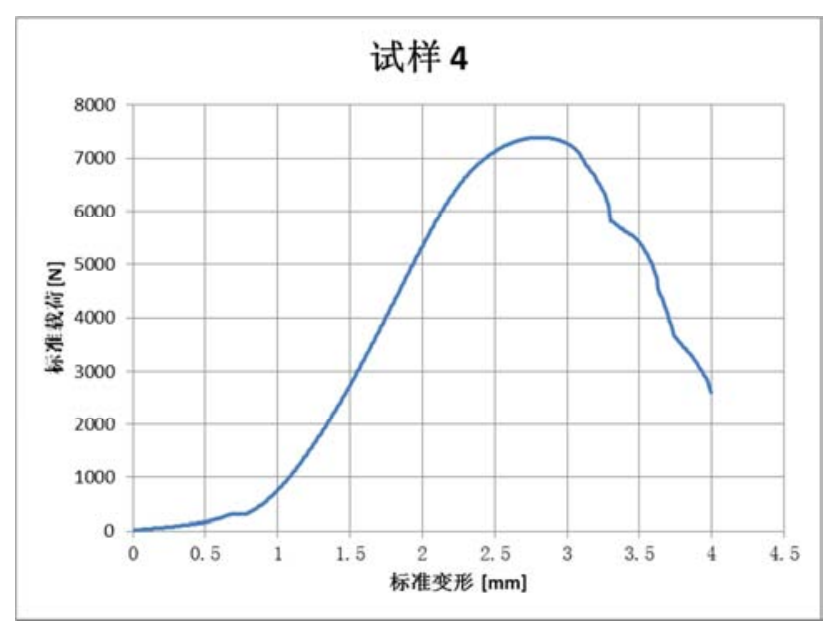

(d)试件4

图1 面内拉伸试件的位移-载荷曲线。
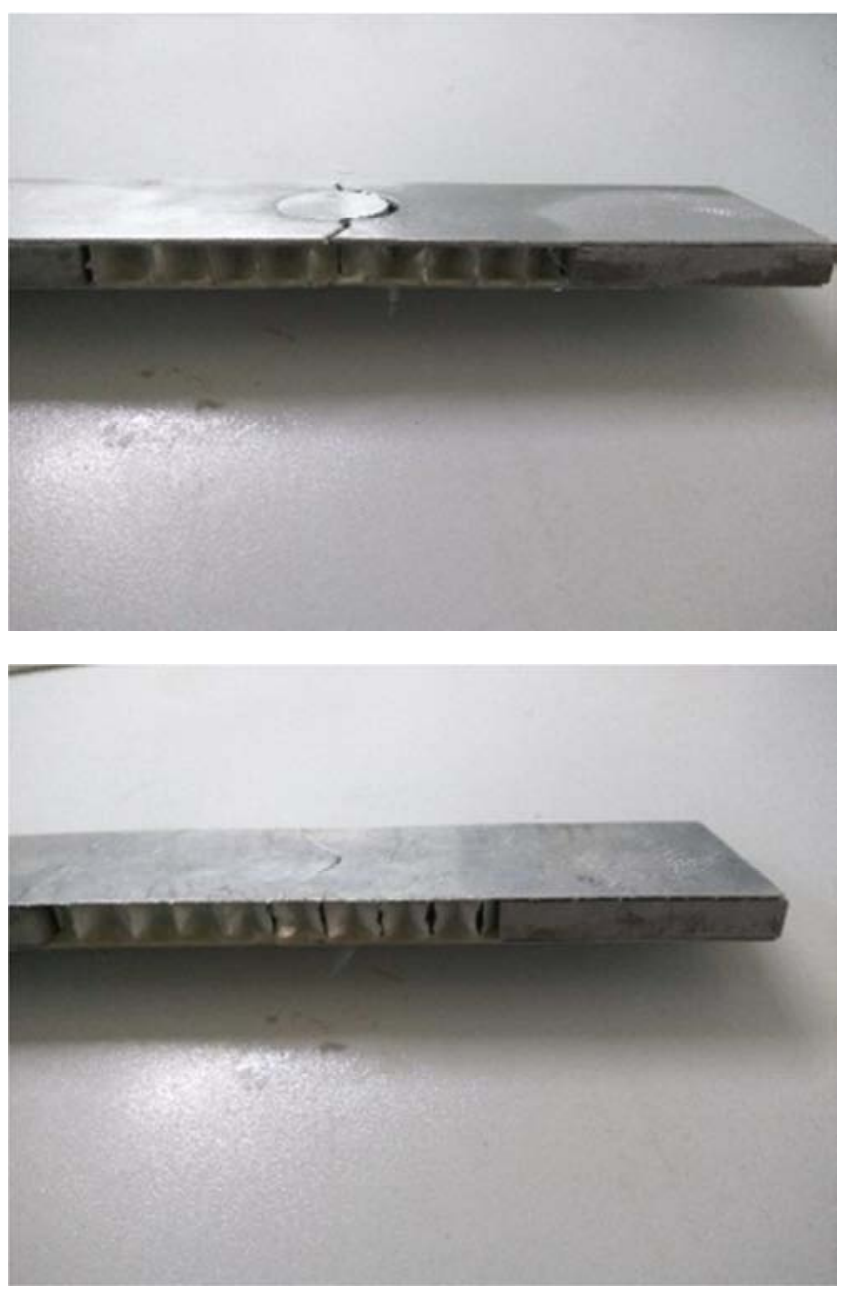

图2 面内拉伸试件失效结果照片。 


\subsection{2. 面内压缩}

用两个压缩平台把试件立夹在中间, 并确保试件与压 缩平台垂直, 然后打开材料试验机开关, 对试件两端施加 压缩载荷，同时，用计算机采集位移载荷数据，直到试件 发生明显的压缩变形为止。所获得的四条位移-载荷曲线 如图3所示。试件发生失效时的试验结果照片如图4所示。

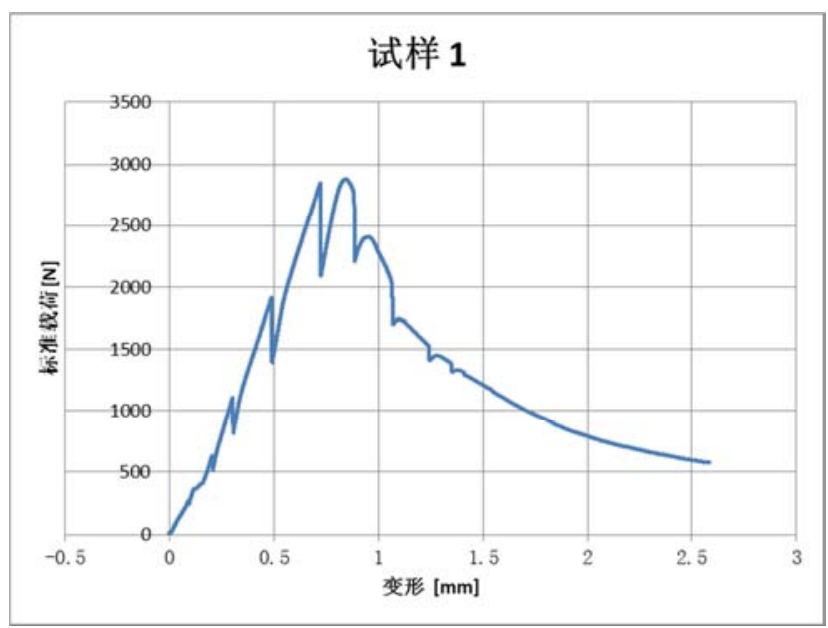

(a)试件1

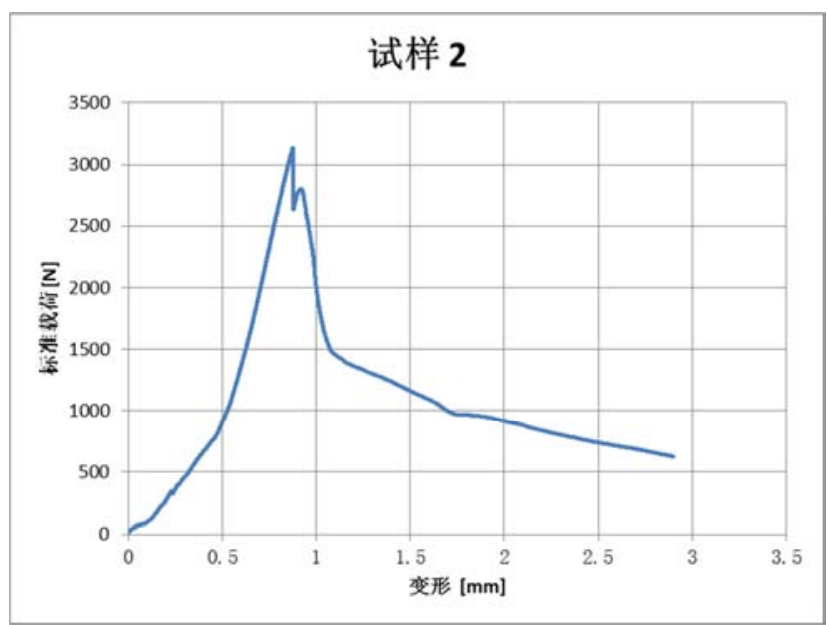

(b)试件2

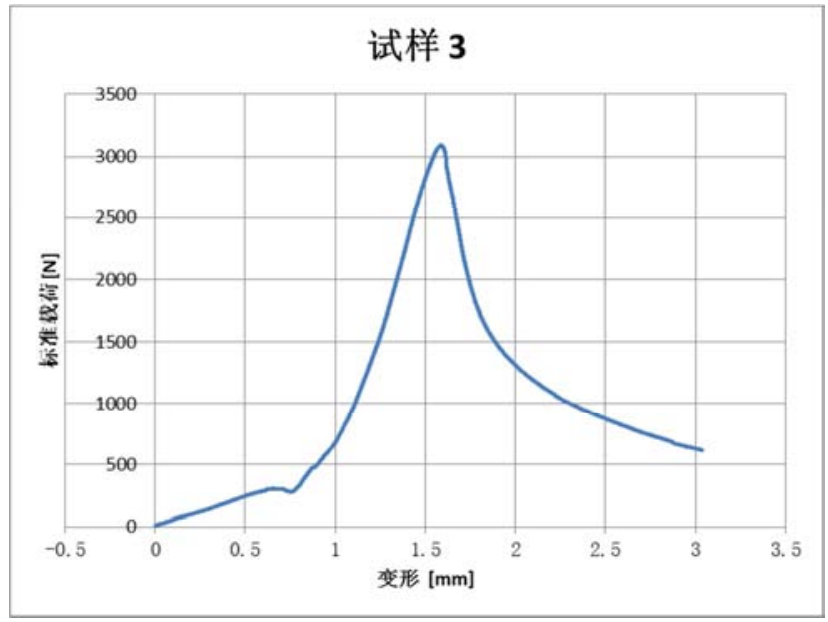

(c)试件3

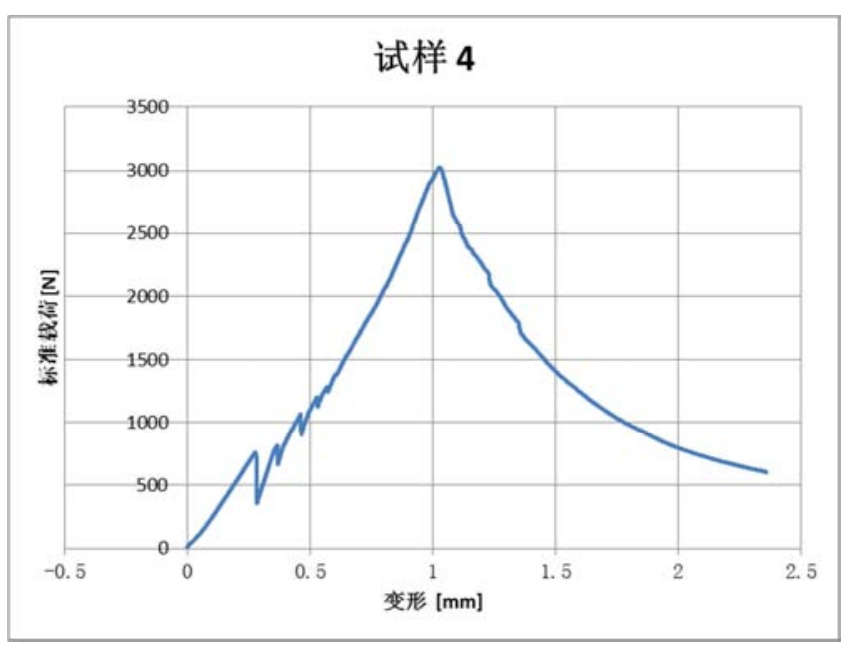

(d)试件4

图3 面内压缩试件的位移-载荷曲线。
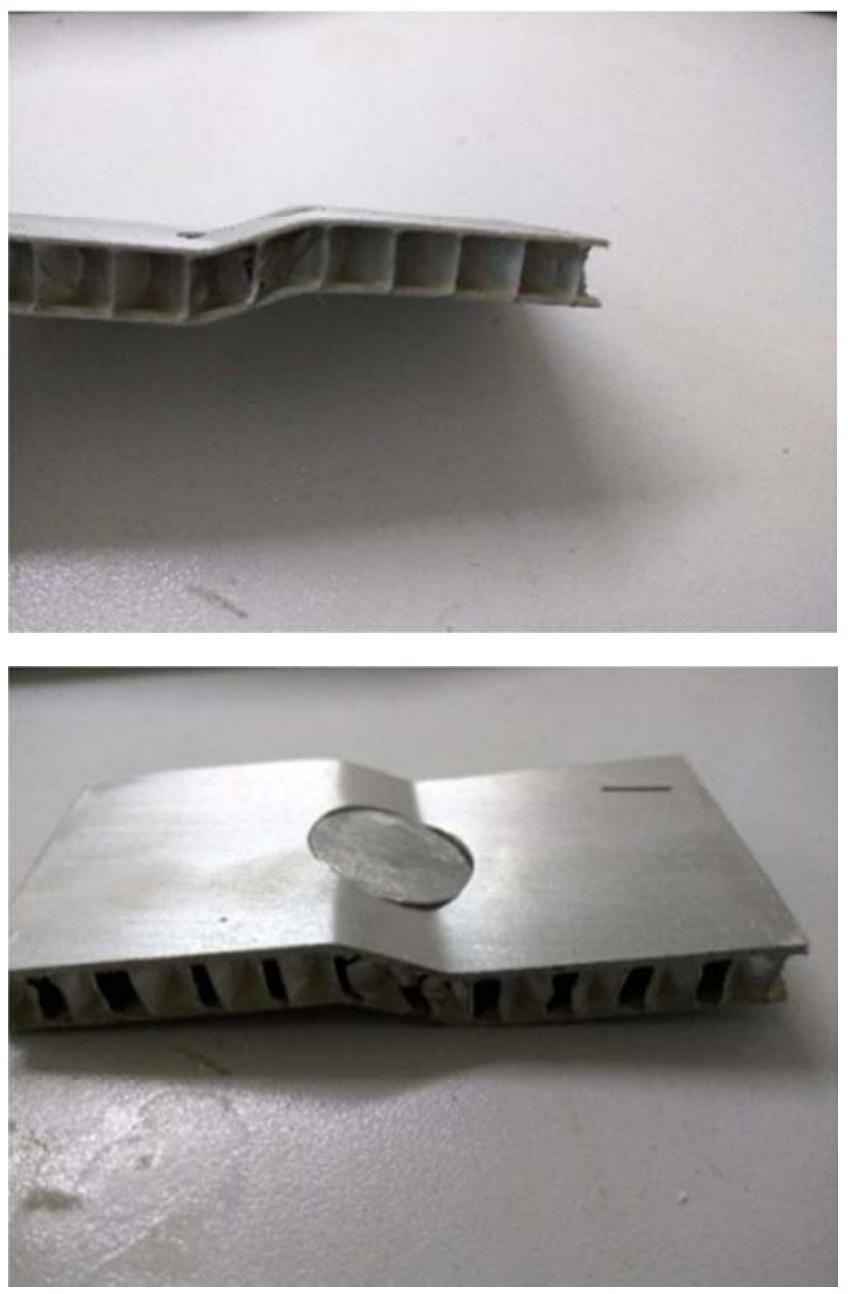

图4 面内压缩试件失效结果照片。

\subsection{3. 三点弯曲}

把试件的下面板放到试验机平台的两个支点上, 并确 保试验机施加的面外压缩载荷的支点对准试件中间, 下方 的两个支点对称分布在两侧, 然后打开材料试验机开关, 对试件中间施加面外压缩载荷, 同时, 用计算机采集位移 
载荷数据, 直到试件发生明显的弯曲变形为止。所得到的 四条位移-载荷曲线如图5所示。试件发生失效时的试验结 果照片如图6所示。

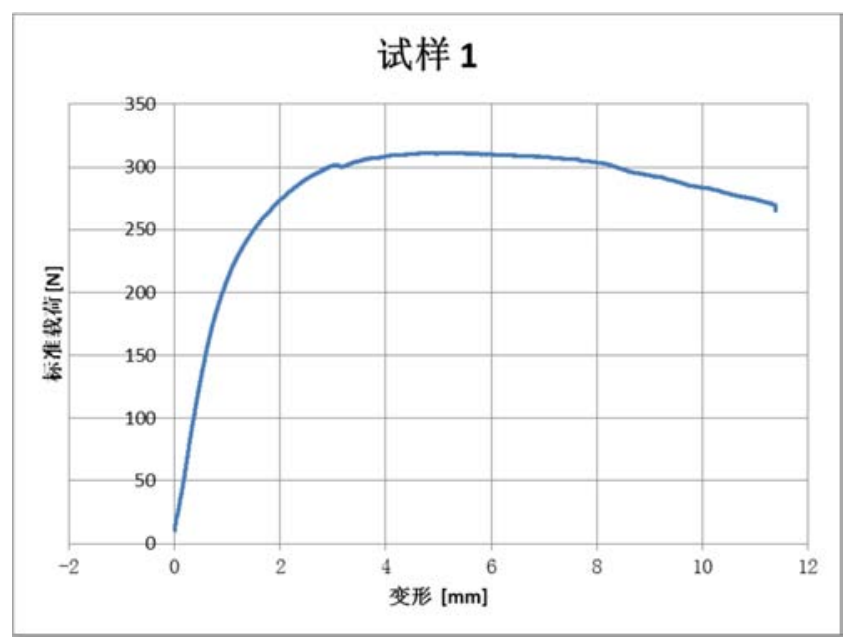

(a)试件1

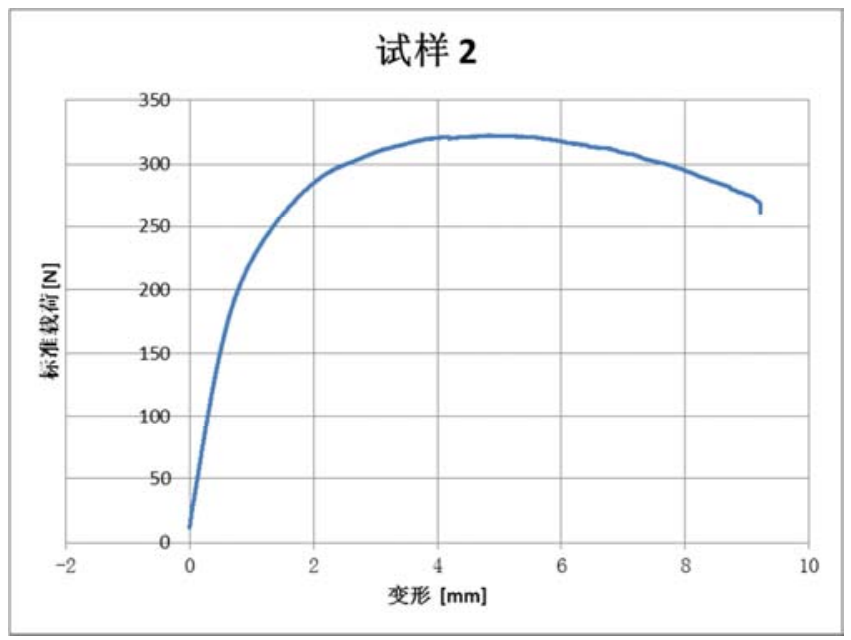

(b)试件 2

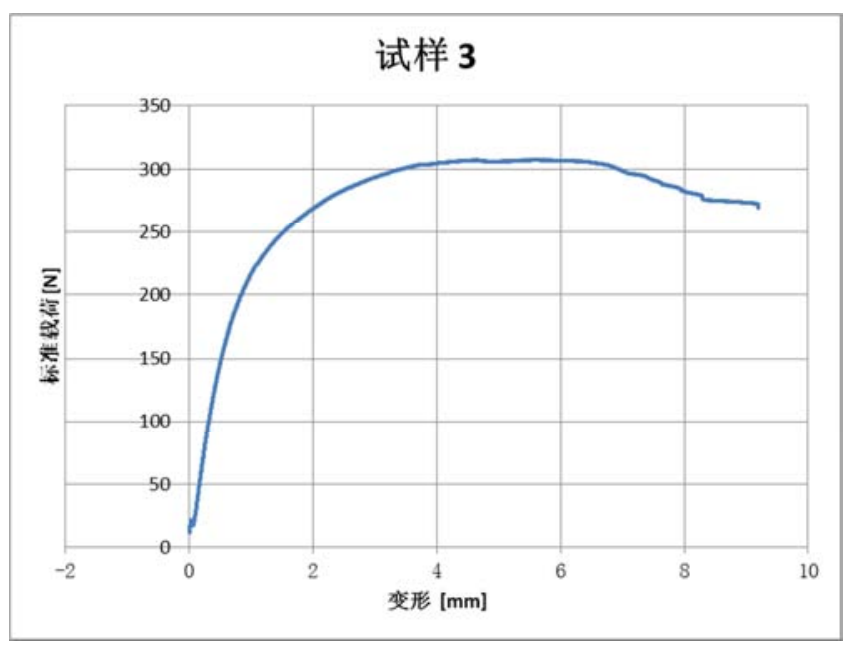

(c)试件3

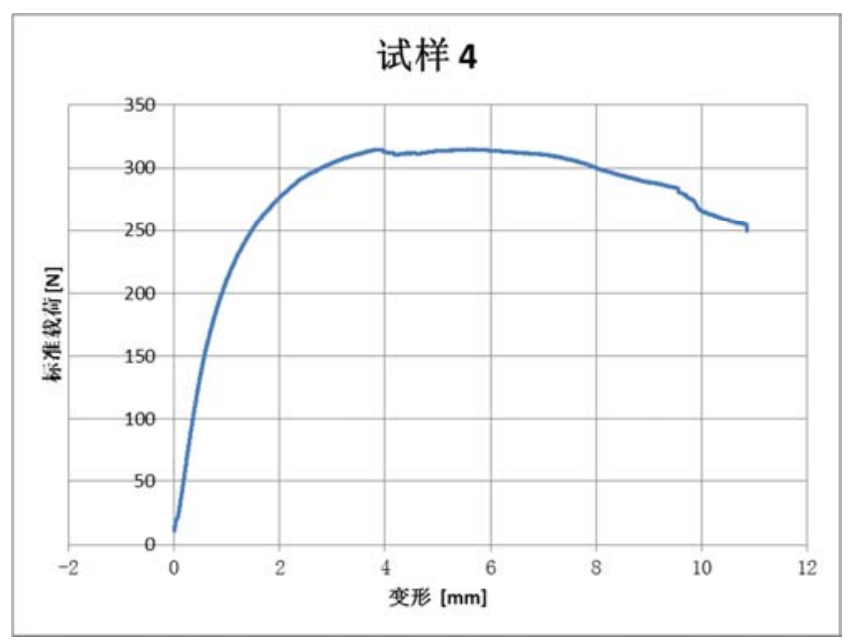

(d)试件4

图5 三点弯曲试件的位移-载荷曲线。
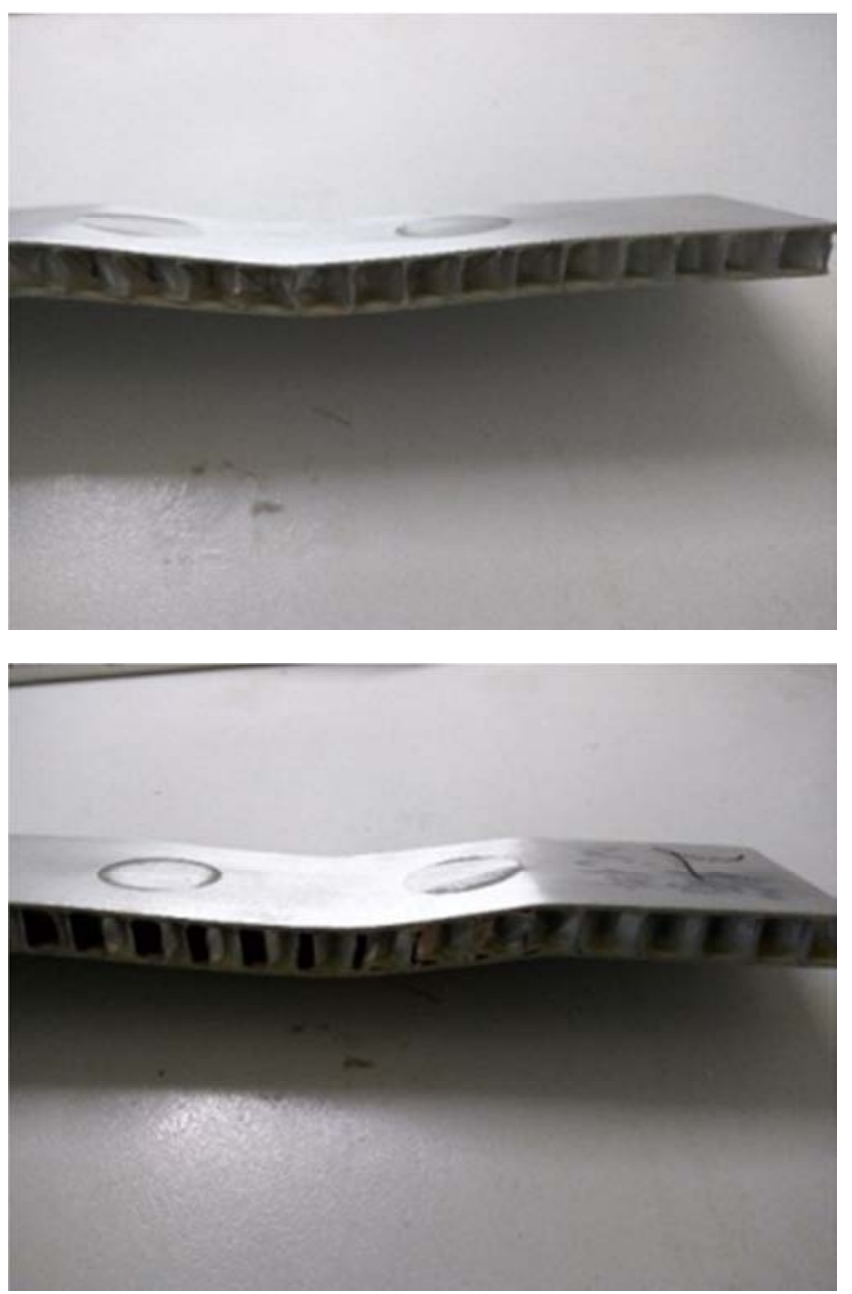

图6 三点弯曲试件失效结果照片。

由上述三种工况下试件发生失效的试验结果照片可 知, 试件失效主要发生在蜂窝壁的上部, 并向蜂窝的壁厚 方向拓展, 这主要是由于蜂窝壁弯曲产生的拉应力导致芯 层失效, 失效部位的拉应力最大 $[15]$ 。 


\section{3. 三种工况有限元模型的修正}

本章在ANSYS软件中分别建立与静力学实验相同的 三种典型工况下含缺陷蜂窝夹芯试件的有限元模型, 再篮 选出相应实验结果中位移与载荷线性相关性最好的半部 分, 得到仿真受载条件, 在ANSYS中的瞬态动力学分析 工具内对有限元模型进行动力学计算, 进而通过修改有限 元模型中的弹性模量使得软件计算的位移结果与实验结 果保持一致, 来对相应的有限元模型进行修正。

\section{1. 拉伸工况有限元模型的修正}

拉伸工况静力学试验得到的四个试件位移-载荷结果 曲线的线性部分如图7所示。舍弃第四个试件的试验数据, 在其它 3 条试验结果曲线上, 分别选取位移为 $0.1 \mathrm{~mm}$, $0.2 \mathrm{~mm}, 0.3 \mathrm{~mm}, 0.4 \mathrm{~mm}$ 时相应载荷的平均值作为ANSYS 中的加载条件。在此条件下, 当把试件材料的弹性模量改 为 $0.987 \mathrm{GPa}$ 时, 试件产生的最大位移为 $0.40131 \mathrm{~mm}$, 与试 验结果的平均值 $0.40156 \mathrm{~mm}$ 近似相等。由此可知该试件材 料的等效弹性模量约为 $0.987 \mathrm{GPa}$ 。

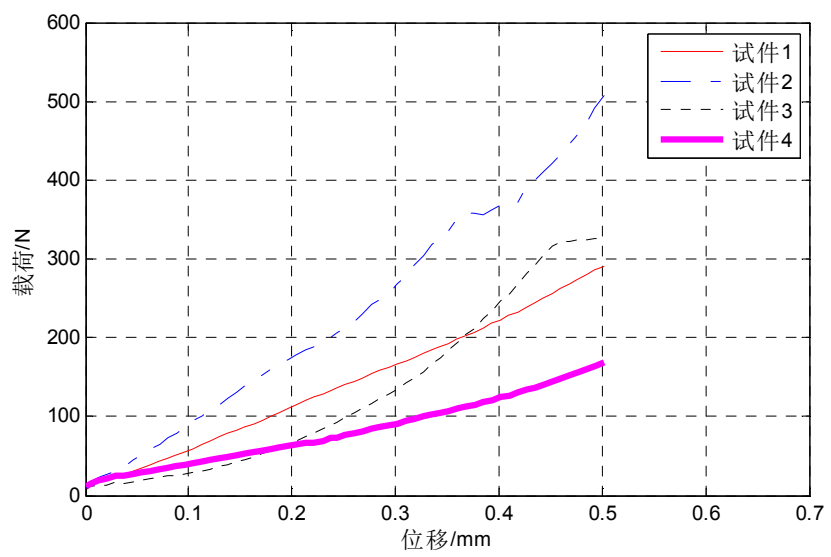

图7 拉伸实验线性阶段位移-载荷曲线。

\section{2. 压缩工况有限元模型的修正}

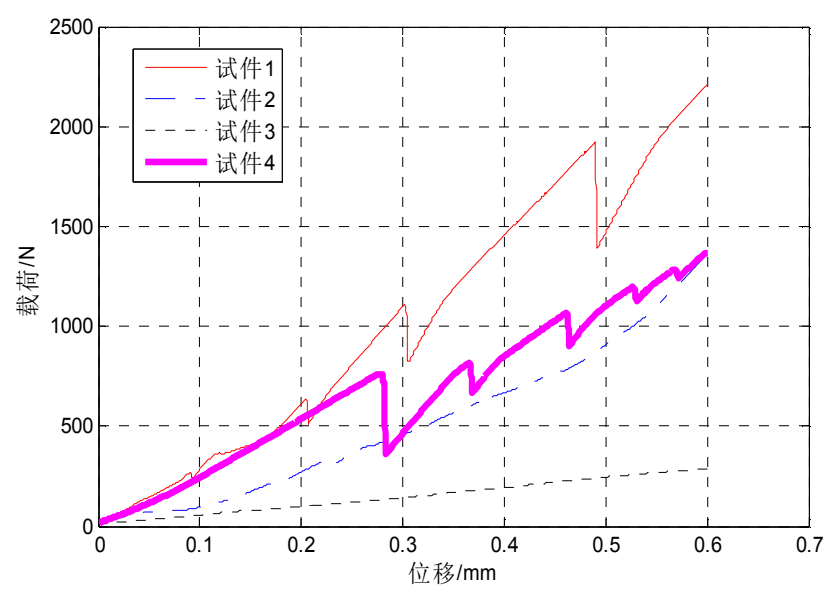

图8 压缩实验线性阶段位移-载荷曲线。

压缩工况静力学试验得到的四个试件位移-载荷结果 曲线的线性部分如图8所示。舍弃第一和第三个试件的试
验数据, 在其它 2 条试验结果曲线上, 分别选取位移为 $0.3 \mathrm{~mm}, 0.4 \mathrm{~mm}, 0.5 \mathrm{~mm}, 0.6 \mathrm{~mm}$ 时相应载荷的平均值作为 ANSYS中的加载条件。在此条件下, 当把试件材料的弹 性模量改为 $3.448 \mathrm{GPa}$ 时, 试件产生的最大位移为 $0.60009 \mathrm{~mm}$, 与试验结果的平均值 $0.600145 \mathrm{~mm}$ 近似相等。 由此可知该试件材料的等效弹性模量约为 $3.448 \mathrm{GPa}$ 。

\section{3. 三点弯曲工况有限元模型的修正}

三点弯曲工况静力学试验得到的四个试件位移-载荷 结果曲线的线性部分如图9所示。在4条试验结果曲线上, 分别选取位移为 $0.1 \mathrm{~mm}, 0.2 \mathrm{~mm}, 0.3 \mathrm{~mm}, 0.4 \mathrm{~mm}$ 时相应载 荷的平均值作为ANSYS中的加载条件。在此条件下，当 把试件材料的弹性模量改为 $10.92 \mathrm{GPa}$ 时, 试件产生的最大 位移为 $0.40072 \mathrm{~mm}$, 与试验结果的平均值 $0.400789 \mathrm{~mm}$ 近似 相等。由此可知该试件材料的等效弹性模量约为 $10.92 \mathrm{GPa}$ 。

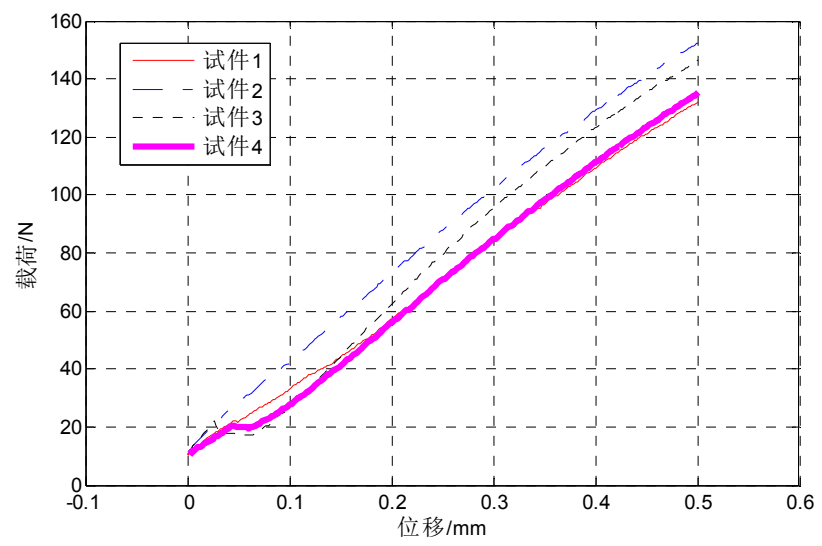

图9 三点弯曲实验线性阶段位移-载荷曲线。

在上述拉伸、压缩、三点弯曲三种工况下试件的等效 弹性模量最大仅为 $10.92 \mathrm{Gpa}$, 远小于铝合金材料的弹性模 量 $(72 \mathrm{GPa})$,与理论推导结果一致[16]。

\section{4. 三种工况的疲劳特性仿真研究}

本章利用ANSYS中的疲劳工具分别分析了三种工况 有限元模型的疲劳特性, 并研究了载荷水平与试件疲劳寿 命的变化规律。选取载荷水平为 $0.55 \sim 0.9$ (共 8 个点) 的仿 真数据, 并采用最小二乘法来拟合疲劳寿命公式。式中, $\mathrm{N}$ 为疲劳寿命, $\mathrm{S}$ 为载荷水平。

\section{1. 拉伸工况}

在不同载荷比条件下, 仿真得到的载荷水平-疲劳寿 命结果数据如下表 1 所示。

表1 拉伸工况不同载荷比的疲劳寿命仿真结果。

\begin{tabular}{llll}
\hline 载荷 & 载荷比 $\mathbf{r}=\mathbf{- 1}$ & 载荷比 $\mathbf{r}=\mathbf{0 . 1}$ & 载荷比 $\mathbf{r}=\mathbf{0 . 2}$ \\
\cline { 2 - 4 } 水平 & 疲劳寿命/次 & 疲劳寿命/次 & 疲劳寿命/次 \\
\hline 0.5 & 799320 & 799940 & 799740 \\
0.55 & 398220 & 398560 & 398440 \\
0.6 & 198390 & 198570 & 198510 \\
0.65 & 105440 & 105520 & 105490 \\
0.7 & 59781 & 59834 & 59816 \\
\hline
\end{tabular}




\begin{tabular}{llll}
\hline 载荷 & 载荷比 $\mathbf{r}=-\mathbf{1}$ & 载荷比 $\mathbf{r}=\mathbf{0 . 1}$ & 载荷比 $\mathbf{r}=\mathbf{0 . 2}$ \\
\cline { 2 - 4 } 水平 & 疲劳寿命/次 & 疲劳寿命/次 & 疲劳寿命/次 \\
\hline 0.75 & 33858 & 33902 & 33887 \\
0.8 & 15735 & 15756 & 15749 \\
0.85 & 7312.4 & 7323 & 7319.5 \\
0.9 & 4023.3 & 4026.8 & 4025.6 \\
\hline
\end{tabular}

载荷比分别为 $-1,0.1,0.2$ 时, 试件的疲劳载荷分别 为 $5424 \mathrm{~N} 、 12052 \mathrm{~N} 、 13559 \mathrm{~N}$; 拟合所得的疲劳寿命近似公 式分别为:

$$
\begin{gathered}
\ln (N)=-13.082 * S+20.103 \\
\ln (N)=-13.08 * S+20.103 \\
\ln (N)=-13.081 * S+20.103
\end{gathered}
$$

\section{2. 压缩工况}

在不同载荷比条件下, 仿真得到的载荷水平-疲劳寿 命结果数据如下表 2 所示。

表2 压缩工况不同载荷比的疲劳寿命仿真结果。

\begin{tabular}{llll}
\hline 载荷 & 载荷比 $\mathbf{r}=\mathbf{- 1}$ & 载荷比 $\mathbf{r}=\mathbf{0 . 1}$ & 载荷比 $\mathbf{r}=\mathbf{0 . 2}$ \\
\cline { 2 - 4 } 水平 & 疲劳寿命/次 & 疲劳寿命/次 & 疲劳寿命/次 \\
\hline 0.5 & 799840 & 799680 & 799630 \\
0.55 & 398500 & 398410 & 398380 \\
0.6 & 198540 & 198500 & 198480 \\
0.65 & 105510 & 105490 & 105480 \\
0.7 & 59825 & 59811 & 59807 \\
0.75 & 33894 & 33883 & 33880 \\
0.8 & 15753 & 15747 & 15745 \\
0.85 & 7321.2 & 7318.5 & 7317.6 \\
0.9 & 4026.2 & 4025.3 & 4025 \\
\hline
\end{tabular}

载荷比分别为 $-1,0.1,0.2$ 时, 试件的疲劳载荷分别 为 $5295 \mathrm{~N} 、 11767 \mathrm{~N} 、 13238 \mathrm{~N}$; 拟合所得的疲劳寿命近似公 式均为:

$$
\ln (N)=-13.081 * S+20.103
$$

\section{3. 三点弯曲工况}

在不同载荷比条件下，仿真得到的载荷水平-疲劳寿 命结果数据如下表 3 所示。

表3 三点弯曲工况不同载荷比的疲劳寿命仿真结果。

\begin{tabular}{llll}
\hline 载荷 & 载荷比 $\mathbf{r}=\mathbf{- 1}$ & 载荷比 $\mathbf{r}=\mathbf{0 . 1}$ & 载荷比 $\mathbf{r}=\mathbf{0 . 2}$ \\
\cline { 2 - 4 } 水平 & 疲劳寿命/次 & 疲劳寿命/次 & 疲劳寿命/次 \\
\hline 0.5 & 798810 & 799920 & 799690 \\
0.55 & 397940 & 398540 & 398420 \\
0.6 & 198240 & 198570 & 198500 \\
0.65 & 105360 & 105520 & 105490 \\
0.7 & 59737 & 59831 & 59813 \\
0.75 & 33822 & 33900 & 33884 \\
0.8 & 15717 & 15755 & 15748 \\
0.85 & 7303.6 & 7322.6 & 7318.8 \\
0.9 & 4020.4 & 4026.6 & 4025.4 \\
\hline
\end{tabular}

载荷比分别为 $-1,0.1,0.2$ 时, 试件的疲劳载荷分别 为 $2516 \mathrm{~N} 、 5590 \mathrm{~N} 、 6289 \mathrm{~N}$; 拟合所得的疲劳寿命近似公式 分别为:

$$
\begin{gathered}
\ln (N)=-13.083 * S+20.102 \\
\ln (N)=-13.081 * S+20.103 \\
\ln (N)=-13.081 * S+20.103
\end{gathered}
$$

\section{4. 检验疲劳寿命公式}

为了验证上述公式的准确性, 将 $S=0.5$ 代入上述九个 公式, 可求得疲劳寿命近似公式的计算值与软件仿真值的 相对误差均在 $2.992 \%$ 以内, 且相关系数均近似等于 1 , 说 明上述疲劳寿命近似公式都较为准确。

\section{5. 结论}

本文提出了一种基于静力学实验与数值仿真相结合 的含缺陷铝蜂窝三明治结构疲劳分析方法, 巧妙地避开了 繁琐复杂的疲劳实验, 能大幅提高对复杂结构疲劳特性的 分析效率, 同时还可降低成本。形成如下结论:

（1）含缺陷蜂窝三明治结构的抗压和抗拉的承载能 力都远大于其抗弯曲承载能力。且蜂窝芯子的缺陷能大幅 降低结构强度, 并使得结构在芯格缺陷的薄弱部位处发生 失效。

（2）考虑到存在实验试件加工误差、试件材料性能 误差、力学实验误差以及蜂窝芯与基板之间的胶水等难以 预测和测量的误差, 导致试件与相应的有限元模型无法完 全相同。因此在修正有限元模型之后, 试件的等效杨氏模 量与普通铝合金存在较大区别。

（3）在相同的载荷类型、材料、载荷比条件下，试 件的载荷水平其疲劳寿命的自然对数之间具有很强的线 性关系，且在材料相同的条件下，即使试件的载荷比和工 况不同, 根据数据拟合得到的疲劳寿命公式中的系数几乎 一致，这表明疲劳寿命公式主要与试件的材料有关。

\section{参考文献}

[1] Barnett D M, Rawal S P. Multifunctional Structures technology experiment on Deep Space 1 mission [J]. Electro-mechanical Engineering, 2001, 1 (1): 13-18.

[2] Joel S, et al. Modular and multifunctional system in the new millennium Program [C]// Reno: AIAA 34th Aerospace Science Meeting and Exhibit, NV, 1996: AIAA Paper 96-0702.

[3] Nathan E H , Daniel R M. Design and testing of Multifunctional structure concept for spacecraft [R]. Denver: Lockheed Martin Astronautics Co, AIAA-2000-1555, 2000.

[4] 曹莉,介党阳,熊楚杨,徐嘉. 多功能结构在固体火箭上的应 用分析 [J].导弹与航天运载技术，2013，(04): 38-42。

[5] Belingardi G, Martella P, Peroni L. Fatigue Analysis of Honeycomb-Composite Sandwich Beams [J]. Composites Part A: Applied Science and Manufacturing, 2007, 38 (4): 1183-1191. 
[6] S. Belouettar, A. Abbadi, Z. Azari, R. Belouettar, et al. Experimental investigation of static and fatigue behaviour of composites honeycomb materials using four point bending tests [J]. Composite Structrues, 2009, 87 (3), 265-273.

[7] Soni SM, Gibson RF, Ayorinde EO. The influence of subzero temperatures on fatigue behavior of composite sandwich structures [J]. Composite Science Technology, 2009, 69 (6), 829-838.

[8] 孔祥皓. 含缺陷金属蜂窝夹层结构的力学性能分析与失效 评估研究[D].哈尔滨：哈尔滨工业大学, 2010:61。

[9] Costantino Menna, Alberto Zinno, Domenico Asprone. Andrea Prota Numerical assessment of the impact behavior of honeycomb sandwich structures [J]. Composite Structures, 2013.106(Complete): 326-339.

[10] Figueroa E, Shafiq B, de la Paz I. Creep to failure and cyclic creep of foam core sandwich composites in seawater [J]. JOURNAL OF SANDWICH STRUCTURES \& MATERIALS, 2013, 15 (6): 657-670.
[11] 赵鹏成. 含缺陷复合材料蜂窝夹层结构力学性能研究 [D]. 哈尔滨：哈尔滨工程大学,2009: 33。

[12] 柴子龙. 复合材料蜂窝夹芯结构疲劳行为研究 [D]. 哈尔滨: 哈尔滨工业大学,2016:66。

[13] 王琦,童国权,陈峰,马振武,杨钦金金. 缺陷对高温合金蜂窝板 弯曲力学性能的影响 [J].航空制造技术, 2017, (1/2):106-111。

[14] 马铭泽,姚卫星,陈炎. 蜂窝夹芯板疲劳研究进展 [J].航空工 程进展, 2019, 10(02):154-162。

[15] 刘红影,张伟,徐佳,刘海金蜂窝复合板疲劳分析[J].玻璃钢/ 复合材料增刊, 2012:111-116。

[16] 高星鹏. 含缺陷高温合金蜂窝板力学性能研究[D].南京: 南 京航空航天大学，2018：26。 